\title{
Research on Popularization and Promotion of Tai Chi Courses in Colleges and Universities
}

\author{
Chen En \\ College of Physical Education, Jimei University, Fujian, China, 361009
}

Keywords: college students; physical and mental health; Tai Chi; value

\begin{abstract}
According to various factors affecting the physical and mental health of college students in the new era, combined with the movement principle and exercise characteristics of Tai Chi, the value of Tai Chi in colleges and universities is discussed. The role of sports in mental health has also been fully confirmed. Regular participation in physical exercise can relieve psychological stress and anxiety, and cultivate perseverance and overcome the difficulties of perseverance and healthy personality. Therefore, to guide college students to establish the concepts of "Health First" and "Lifetime Sports" and to develop the habit of physical exercise to regulate physical and mental health are the requirements for comprehensive quality education and effective ways to enhance the physical and mental health of college students.
\end{abstract}

\section{Introduction}

At present, the physical and mental health of college students in China is worrisome. For this reason, colleges and universities have established corresponding adjustment and emergency mechanisms. However, the effect is still not optimistic. In order to further improve the health status of college students, we must also do all kinds of efforts. Institutions can play an active role in the training of talents in sports, through the popularization of traditional sports to achieve the cultivation and shaping of college students' body and mind. Sports are characterized by hardship, fatigue, intenseness, tension, confrontation, and competitiveness. When participating in physical exercise, there is always a strong emotional experience and a clear will to work hard[1].

\section{Factors that Affect the Physical and Mental Health of College Students}

\subsection{Psychological factors.}

There are many reasons for the current deterioration of the physical condition and mental health of undergraduates, mainly including:

(1) Self-perception confusion. They lack the correct understanding and positioning of themselves, causing the confusion of self-identity, which leads to confusion in the direction of self-image development. There is a lack of profound reflection on his own development status, starting point, goals, and approaches; he is not at ease with the intersection of individual independence and social themes, forms, and styles.

(2) Affective factors. In the materially advanced market economy environment, contemporary college students are affected by multiculturalism and multi-layered morality, and emotional venting is more adequate than any generation. Chinese medicine believes that people's seven passions and six desires can cause illness, that is, excessive happiness, anger, worry, thought, sadness, fear, and panic can cause corresponding diseases. Modern scientific research also shows that long-term depression, anxiety or major mental stimulation can cause the body's immune function to decline and enter sub-health (between the "third state of health and unhealthy state")[2].

(3) Will factors. The intensification of social competition, the busy academic tasks, the increased personal pressure, the physical fatigue after learning, the lack of interest in sports, poor physical fitness, unwillingness to exercise, the lack of attention to physical fitness, and the lack of hard-working spirit. 
(4) Sexual psychological factors. At present, college students are living in the environment of media diversification, campus love, cohabitation, and on the other hand, they are exposed to the lack of sex education. Students' confusion in the process of love and sex cannot be properly guided. The desire, anxiety and depression caused by sexual impulses have become an important factor affecting students' physical and mental health [3].

\subsection{Social factors.}

One is the negative impact of social values. The various moral and spiritual changes that occur in society have caused the confusion of college students' values and their emotional distress.

The second is heavy employment pressure. At present, the employment situation is grave and parents have too high expectations, which makes the employment anxiety of contemporary college students occupy the spiritual space, all kinds of contradictions gather, long-term backlog, resulting in adverse reactions to physical and mental health.

\subsection{School management factors.}

At present, there are many bad tendencies among students. Such as campus consumption comparison phenomenon, violence phenomenon, exam cheating and so on. Various fame and money relationships have a great influence on college students. However, in terms of political and ideological education and administrative management, the school has not done enough work and has not fundamentally solved these problems, which has become another factor that affects the physical and mental health of college students. School management factor formula is as follows.

$$
p_{i j}=x_{i j} / \sum_{i=1}^{m} x_{i j}
$$

\section{The Value of Tai Chi}

\subsection{A treasure in traditional sports.}

Tai Chi is a precious asset of the Chinese nation. It is not only an ancient boxing technique that emphasizes both fitness and combat, but also contains profound Chinese classical philosophy. Tai Chi is set as a compulsory subject in physical education. Popularizing Tai Chi in college students is not only beneficial to physical fitness but also self-defense and self-defense. It is also conducive to the inheritance of Chinese Wushu culture and has been welcomed by most students. Through Tai Chi teaching, students have a better understanding of our national traditional sports culture, national traditional sports concept, and types of Tai Chi and boxing. Among them, 46\% said that they will choose Tai Chi as their future fitness. means [4].

\subsection{Obviously perfect fitness.}

Tai Chi has absorbed theories of meridians, acupuncture points, blood, guidance, and Tibetan elephants of traditional medicine. It requires breathing and movement to cooperate with each other, "transmitting with air," "transporting with your body," and "breathing with your body." Take the waist as the axis, drive the limbs, turn the spine and turn back, and spirally wind up. Imagine the power transmission, follow the classics, pass through the points, spread the whole body, pass the internship with the pulse, promote blood running, and clear the meridians, Play a fitness role, that is, boxing on what is said, "If you ask what is the end of intention, Yishou Yannian is not old." Therefore, it has a greater role in health care and has attracted the attention of the medical community. It has now become one of the important forms of medical sports in China.

\subsection{Unique attack.}

Tai Chi has integrated and absorbed the boxing techniques of the Ming Dynasty, and in particular has drawn Qi Jiguang's 32-style Changquan, combined with ancient guiding techniques, the technique of breathing Qigong, the meridian theory of traditional Chinese medicine, and the simple and plain dialectical materialism theory of yin and yang. Daoism and Tai Chi Eight Seal 
Theory are the philosophical foundation of Taijiquan. Tai Chi contains a wealth of Chinese traditional culture and philosophy. Taijiquan is based on the basic techniques of "stretching, squeezing, squeezing, pressing, mining, leaning, elbow, leaning, advancing, retreating, caring, expecting, and deciding”. The movement requires meditation and intentions to guide movements, and the movements and breathing are closely coordinated. The movements must be moderately comfortable, gentle and slow, the body should remain comfortable and natural, impartial, and the movements should be continuous, soft and natural, and the movement should be curved. Hysteresis, at the same time with the waist as the axis, with the up and down, the whole body form a whole.

\section{Psychological Adjustment and Character Cultivation of Tai Chi}

\subsection{Adjust college students' psychological status.}

College students, as mental workers of the present and the future, have long been attentive. The pace of modern social life is accelerating, the sense of competition is gradually strengthened, and the pressure of survival is everywhere, resulting in the nervous system being in a state of inhibition for a long time, and it is easy to produce anxiety, fear, neurasthenia, and even personality disorders. Tai Chi moves $\mathrm{Xu}$, gentle, soft, smooth, round, light and spiritual. The emotion relaxes as the practice moves like a cloud, and persists in exercise is energetic, relieving or eliminating the morbid state caused by irritability, insomnia, forgetfulness and other undesirable mental states. There is no competition and psychological pressure in Taijiquan. In addition, the spiritual realm of Taijiquan is "clean and inaction", "going to desires, and simple things". Over time, it can form a purification and special state of the soul, and improve the impetuous and utilitarian mentality. Changes in the scores of Taijiquan's regulatory functions are shown below.

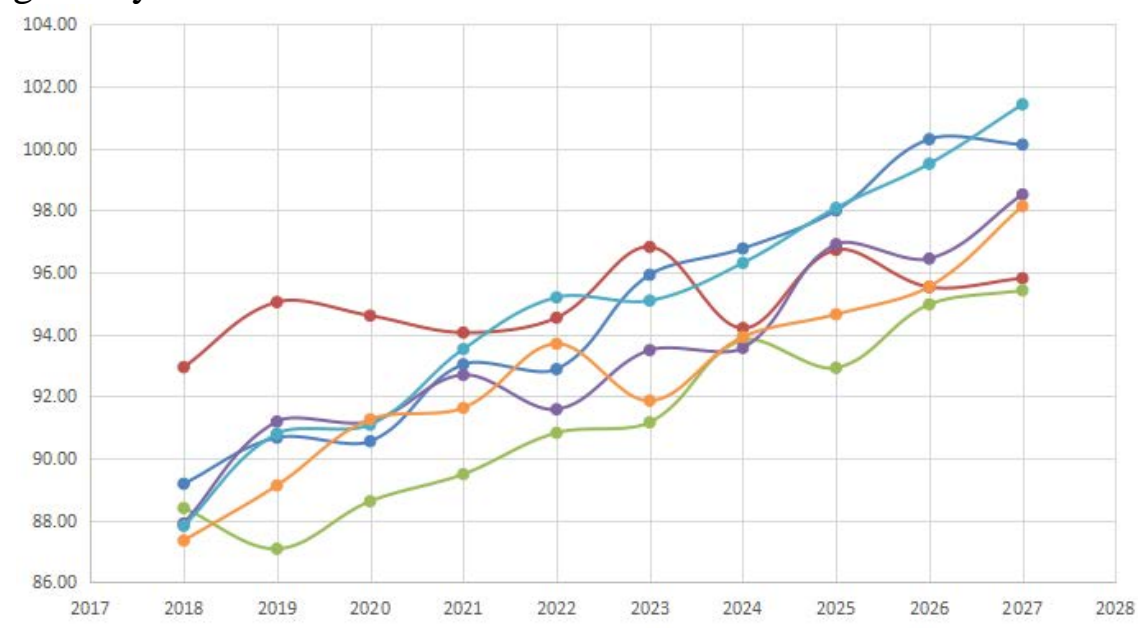

Fig.1 Changes in the scores of Taijiquan's regulatory functions

\subsection{Instill college students' ideological quality.}

Tai Chi Chuan emphasizes the slow rhythm, calm and calmness, and does not take the initiative and practice rituals. It can train Wu Xide and cultivate college students' noble sentiments. The practice of Tai Chi is not a day's work. The process of martial arts is boring and boring. It requires perennial unremitting efforts to cultivate the spirit of hard-working and courageous students in overcoming difficulties. Mastering the Taijiquan movement requires a thousand times of imitation, practice, contemplation, reflection, and comprehension. It can cultivate the enterprising spirit of university students who are never complacent and strive for excellence. The potential skill of Taijiquan has given the practitioners fighting skills, and at the same time it has created the courageous, strong and persevering qualities of college students.

\section{Summary}

With the establishment of the "health first" guiding ideology, the content of teaching should shift 
to high quality, light weight and negative aspects. The value of Tai Chi exercise in college sports teaching is more and more obvious. It is not only the precious cultural heritage of the Chinese nation, but also meets the needs of modern higher education. It has a moderate amount of exercise and does not require too much space and equipment. It has a remarkable effect in enhancing physical fitness and promoting physical and mental health. It is a sporting exercise that can be practiced at any time. Learning Taijiquan at the university level is not only for physical fitness but also for the psychological health of college students. Therefore, popularizing Tai Chi, a popular national fitness program, is widely used in colleges and universities. It has positive and important values and significance for cultivating qualified trans-century talents.

\section{References}

[1] Daniel Mroz. Technique in exile: The changing perception of taijiquan, from Ming dynasty military exercise to twentieth-century actor training protocol[J]. Studies in Theatre and Performance, 2008,28(2).

[2] Hai Yu. Research on Inherent Laws of Taijiquan Teaching Method[M]. Springer London: 2013-06-15.

[3] Caldwell Karen, Emery Lisa, Harrison Mandy, Greeson Jeffrey. Changes in mindfulness, well-being, and sleep quality in college students through taijiquan courses: a cohort control study. [J]. Journal of Alternative \&amp; Complementary Medicine,2011,17(10).

[4] Komelski Matthew F, Miyazaki Yasuo, Blieszner Rosemary. Comparing the health status of U.S. taijiquan and qigong practitioners to a national survey sample across ages. [J]. Journal of Alternative \&amp; Complementary Medicine,2012,18(3). 